\author{
Alba Perez-Gracia \\ Institut de Robòtica i Informàtica Industrial \\ (CSIC-UPC), \\ Llorens i Artigas, 4-6, \\ 08028 Barcelona, Spain; \\ College of Science and Engineering, \\ Idaho State University, \\ 921 South 8th Avenue, \\ Pocatello, ID 83209 \\ e-mail: aperez@iri.upc.edu
}

\title{
Synthesis of Spatial RPRP Closed Linkages for a Given Screw System
}

The dimensional synthesis of spatial chains for a prescribed set of positions can be applied to the design of parallel robots by joining the solutions of each serial chain at the end-effector. This design method does not provide with the knowledge about the trajectory between task positions and, in some cases, may yield a system with negative mobility. These problems can be avoided for some overconstrained but movable linkages if the finite-screw system associated with the motion of the linkage is known. The finite-screw system defining the motion of the robot is generated by a set of screws, which can be related to the set of finite task positions traditionally used in the synthesis theory. The interest of this paper lies in presenting a method to define the whole workspace of the linkage as the input task for the exact dimensional synthesis problem. This method is applied to the spatial RPRP closed linkage, for which one solution exists.

[DOI: $10.1115 / 1.4003846$ ]

\section{Introduction}

Synthesis of parallel robots has focused mainly on type or structural synthesis, using group theory, screw theory, or geometric methods, see, for instance, Refs. [1-3]. Dimensional synthesis examples exist, mainly for optimizing performance indices [4-6] or for reachable workspace sizing [7-9], see also Ref. [10] for a comprehensive approach.

The dimensional synthesis of spatial serial chains for a prescribed set of positions can be used for the design of parallel robots by synthesizing all supporting legs for the same set of positions. There are a few examples of finite-position dimensional synthesis of parallel robots in the literature, most of them doing partial synthesis. Wolbrecht et al. [11] performed synthesis of 3-RRS, 4-RRS, and 5-RRS symmetric parallel manipulators; Kim and Tsai [12] and Rao [13] solved the partial kinematic synthesis of a 3-RPS parallel manipulator. This method has been successfully applied mainly to special parallel systems with imposed symmetry. In general, the method does not allow the control of the final trajectory of the parallel system; in the most extreme cases, it may yield a system with negative mobility that can be assembled at each task positions but cannot be driven from task position to task position.

The kinematic mapping is used for the synthesis of planar and spherical linkages in order to state design equations and to provide a tool for visualizing the workspace and trajectories of the linkage. See Ref. [14] and more recent applications by Hayes [15], Schröcker [16], and Wu and Ge [17]. For spatial motion, Study's kinematic mapping is used to obtain simplified equations for analysis and synthesis, see Refs. $[18,19]$. However, the kinematic image for the spatial motion is a six-dimensional quadric and that makes the visualization of workspaces and trajectories difficult for the designer.

In this paper, the workspace of the linkage is visualized as a set of finite screws corresponding to the set of finite displacements of the end-effector. One interesting question is whether the finitescrew surfaces generated by a set of task positions can give any information for the synthesis of parallel robots and, in particular, of some overconstrained closed linkages. Using Parkin's defini-

Contributed by the Mechanisms and Robotics Committee of ASME for publication in the Journal of Mechanisms and Robotics. Manuscript received May 11, 2010; final manuscript received February 15, 2011; published online April 11, 2011. Assoc. Editor: Andrew P. Murray tion for pitch [20], the screws corresponding to finite displacements of some linkages can form screw systems. Huang [21] showed that the single RR chain forms a finite-screw system of third order; however, the set of finite displacements of the coupler of the Bennett linkage form a cylindroid, which is a general twosystem of screws [22]. Baker [23] also studied the motion of the Bennett linkage. Perez and McCarthy [24] used two arbitrary displacements to generate the cylindroid of finite screws associated to the Bennett linkage in order to perform dimensional synthesis.

In this paper, the focus is on the simplest of the overconstrained linkages, the closed spatial RPRP linkage. This linkage is one of the Delassus linkages, later studied by Waldron [25] and Baker [26]. Recently, Huang [27] showed that the set of screws corresponding to displacements of this linkage forms a two-screw system. We use this result in order to synthesize RPRP linkages with positive mobility and for a given shape of the screw system of relative displacements. In order to do so, we state the design equations using the Clifford algebra of dual quaternions [28]. The dual quaternion expression can be easily related to the screw system and it is also used to assign the magnitude to the screws in order to obtain the correspondence between the screw system and the trajectory of the end-effector. The design yields a single RPRP linkage.

\section{Clifford Algebra Equations for the Synthesis}

The approach used in this paper for stating design equations is based on the method of Lee and Mavroidis [29]. They equate the forward kinematics of a serial chain to a set of goal displacements and consider the Denavit-Hartenberg parameters as variables. A more efficient formulation for our purposes consists of stating the forward kinematics of relative displacements using the even Clifford subalgebra of the projective space $C^{+}\left(P^{3}\right)$, also known as dual quaternions [28].

The Plücker coordinates $\mathbf{S}=(\mathbf{s}, \mathbf{c} \times \mathbf{s})$ of a line can be identified with the Clifford algebra element $\mathbf{S}=\mathbf{s}+\varepsilon \mathbf{c} \times \mathbf{s}$, with $\varepsilon^{2}=0$. Similarly, a screw $\mathrm{J}$ becomes the element $\mathrm{J}=(1+\mu \varepsilon) \mathrm{S}$, where $\mu$ is the pitch. Using the Clifford product, we can compute the exponential of the screw $(\theta / 2) \mathrm{J}$,

$$
e^{(\theta / 2)\lrcorner}=\left(\cos \frac{\theta}{2}-\frac{d}{2} \sin \frac{\theta}{2} \varepsilon\right)+\left(\sin \frac{\theta}{2}+\frac{d}{2} \cos \frac{\theta}{2} \varepsilon\right) \mathrm{S}=\cos \frac{\hat{\theta}}{2}+\sin \frac{\hat{\theta}}{2} \mathrm{~S}
$$




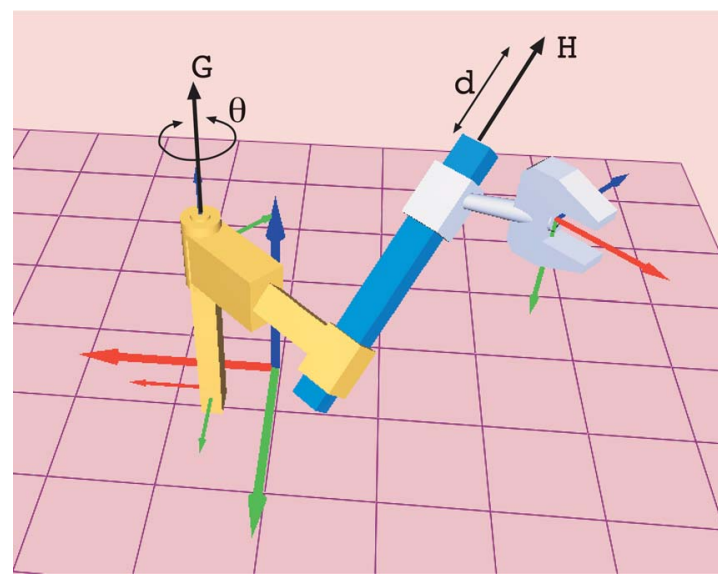

Fig. 1 The RP serial chain

The exponential of a screw defines a unit dual quaternion, which can be identified with a relative displacement from an initial position to a final position in terms of a rotation around and a slide along an axis.

2.1 Forward Kinematics. For a serial chain with $n$ joints, in which each joint can rotate an angle $\theta_{i}$ and slide a distance $d_{i}$, around and along the axis $\mathrm{S}_{i}, i=1, \ldots, n$, the forward kinematics of relative displacements (with respect to a reference position) can be expressed as the composition of Clifford algebra elements corresponding to the motion of each joint. Let $\vec{\theta}_{0}$ and $\vec{d}_{0}$ be the joint parameters of this chain when in the reference configuration, so we have $\vec{\Delta} \hat{\theta}=\left(\boldsymbol{\theta}-\boldsymbol{\theta}_{0}+\left(\boldsymbol{d}-\boldsymbol{d}_{0}\right) \boldsymbol{\varepsilon}\right)$. Then, the movement from this reference configuration is defined by

$$
\begin{aligned}
\hat{Q}(\Delta \vec{\theta})= & e^{\left(\Delta \hat{\theta}_{1} / 2\right) \mathrm{S}_{1}} e^{\left(\Delta \hat{\theta}_{2} / 2\right) \mathrm{S}_{2}} \cdots e^{\left(\Delta \hat{\theta}_{n} / 2\right) \mathrm{S}_{n}}=\left(\cos \frac{\Delta \hat{\theta}_{1}}{2}+\sin \frac{\Delta \hat{\theta}_{1}}{2} \mathrm{~S}_{1}\right) \\
& \left(\cos \frac{\Delta \hat{\theta}_{2}}{2}+\sin \frac{\Delta \hat{\theta}_{2}}{2} \mathrm{~S}_{2}\right) \cdots\left(\cos \frac{\Delta \hat{\theta}_{n}}{2}+\sin \frac{\Delta \hat{\theta}_{n}}{2} \mathrm{~S}_{n}\right)
\end{aligned}
$$

The RPRP linkage has a mobility $M=-2$ using the KutzbachGruebler formula; however, for certain dimensions of the links, it moves with one degree of freedom. Waldron [25] showed that the RPRP linkage will have positive mobility only if both revolute joints are parallel, and the directions of the prismatic joints are symmetric with respect to the plane containing the revolute joints.

The RPRP linkage can be seen as a serial RP chain and a serial PR chain joined at their end-effectors. The RP serial chain consists of a revolute joint followed by a prismatic joint. Figure 1 shows the RP serial chain and Fig. 2 shows a sketch of the RPRP linkage with its axes. In the PR serial chain, the order of the joints in the chain is switched.

For both the RP and PR serial chains, let $G=\mathbf{g}+\varepsilon \mathbf{g}^{0}$ be the revolute joint axis, with rotation $\theta$, and $\mathrm{H}=\mathbf{h}+\varepsilon \mathbf{h}^{0}$ the prismatic joint axis, with slide $d$. Notice that, for synthesis purposes, the location of the slider, given by $\mathbf{h}^{0}$, is irrelevant. The Clifford algebra forward kinematics equations for the RP chain are

$$
\begin{aligned}
\hat{Q}_{\mathrm{RP}}(\Delta \theta, \Delta d)= & \left(\cos \frac{\Delta \theta}{2}+\sin \frac{\Delta \theta}{2} \mathrm{G}\right)\left(1+\varepsilon \frac{\Delta d}{2} \mathrm{H}\right) \\
= & \left(\cos \frac{\Delta \theta}{2}+\sin \frac{\Delta \theta}{2} \mathbf{g}\right)+\varepsilon\left(\frac{\Delta d}{2} \cos \frac{\Delta \theta}{2} \mathbf{h}+\sin \frac{\Delta \theta}{2} \mathbf{g}^{0}\right. \\
& \left.+\frac{\Delta d}{2} \sin \frac{\Delta \theta}{2}(\mathbf{g} \times \mathbf{h}-\mathbf{g} \cdot \mathbf{h})\right)
\end{aligned}
$$

For the PR chain, the only difference is a negative sign in the

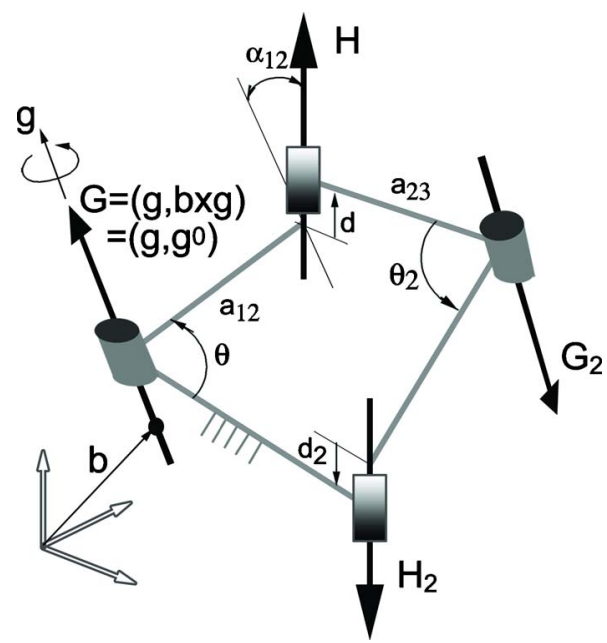

Fig. 2 The RPRP closed linkage

cross product. In Eq. (3), the angle and slide are measured from a reference configuration.

2.2 Design Equations and Counting. Given a set of $m$ task positions expressed as relative displacements, $\hat{P}_{1 j}=\cos \left(\Delta \hat{\phi}_{1 j} / 2\right)$ $+\sin \left(\Delta \hat{\phi}_{1 j} / 2\right) \mathrm{P}_{1 j}, j=2, \ldots, m$, we equate them to the forward kinematics equations in Eq. (2),

$$
\hat{P}_{1 j}=e^{\left(\Delta \hat{\theta}_{1 j} / 2\right) \mathrm{S}_{1}} e^{\left(\Delta \hat{\theta}_{2 j} / 2\right) \mathrm{S}_{2}} \ldots e^{\left(\Delta \hat{\theta}_{n j} / 2\right) \mathrm{S}_{n}}, \quad j=2, \ldots, m
$$

in order to create the design equations.

The result is $8(m-1)$ design equations. The design variables that determine the dimensions of the chain are the $n$ joint axes $S_{i}$, $i=1, \ldots, n$, in the reference configuration. In addition, the equations contain the $n(m-1)$ pairs of joint parameters $\Delta \hat{\theta}_{i j}=\Delta \theta_{i j}$ $+\Delta d_{i j} \varepsilon$, which are also unknown.

For the RP (and similarly for the PR) serial chain, the design equations are

$$
\hat{Q}_{\mathrm{RP}}\left(\Delta \theta^{j}, \Delta d^{j}\right)=\hat{P}_{1 j}, \quad j=2, \ldots, m
$$

The counting of independent equations and unknowns defines the maximum number of arbitrary positions $m$ that can be reached, based only on the type and number of joints of the serial chain, see Ref. [30] for details. Consider a serial chain with $r$ revolute and $p$ prismatic joints. The maximum number of task positions is given by

$$
m=\frac{3 r+p+6}{6-(r+p)}
$$

For serial chains with less than three revolute joints, the structure of semidirect product of the composition of displacements needs to be considered, and the maximum number of rotations $m_{R}$ needs to be calculated too. Assuming that the orientations are given and that both the directions of the revolute joints and the angles to reach the task orientations are known, we can count, in a similar fashion, the number of translations $m_{T}$ that the chain can be defined for.

$$
m_{R}=\frac{3+r}{3-r}, \quad m_{T}=\frac{2 r+p+3}{3-p} .
$$

In order to determine the maximum number of task positions for the RP and PR chains, we apply Eq. (6) to obtain $m=2.5$ task positions. Additional information is obtained using Eq. (7) to compute $m_{R}=2$ task rotations and $m_{T}=3$ task translations. Hence, 
we can define one arbitrary relative displacement and a second relative displacement whose orientation is not general.

\section{Screw System for the RPRP Linkage}

In the context of this paper, a finite-screw surface is a ruled surface in which the lines with their associated pitch correspond to relative displacements. A screw surface will be a screw system if it is closed under addition and scalar multiplication, that is, if every screw of the set can be written as a linear combination of screws belonging to it.

3.1 The Finite-Screw System Generated by the Motion of the RPRP Linkage. The linear combination of two arbitrary screws representing relative displacements forms a two-system known as the cylindroid, which turns out to be the manifold for the relative displacements of the closed 4R linkage. Huang [27], by intersecting the three-systems associated with the finite displacements of the RP and PR dyads, showed that the screw surface of the closed RPRP linkage forms a two-system of a special type, the fourth special type according to Hunt [31], also known as 2-IB [32]. The screws of this system are parallel, coplanar screws whose pitches vary linearly with their distance.

The screw system corresponding to the RPRP linkage can be obtained in a quite straightforward way by using the composition of relative displacements at each joint, as expressed in Eq. (3). Let us denote the resulting displacements

$$
\begin{aligned}
\hat{Q}_{\mathrm{RP}}(\Delta \theta, \Delta d)= & \cos \frac{\widehat{\Delta \psi}}{2}+\sin \frac{\widehat{\Delta \psi}}{2} \mathrm{~S}_{\mathrm{RP}}=\left(\cos \frac{\Delta \psi}{2}-\varepsilon \frac{\Delta t}{2} \sin \frac{\Delta \psi}{2}\right) \\
& +\sin \frac{\Delta \psi}{2}\left(\begin{array}{c}
\frac{\Delta t}{2} \\
1+\varepsilon \frac{\Delta \psi}{\tan \frac{\Delta \psi}{2}}
\end{array}\right) \mathrm{S}_{\mathrm{RP}}
\end{aligned}
$$

where $\Delta \psi$ and $\Delta t$ are the angle about and slide along the screw axis $S_{R P}$ of the resulting relative displacement. Expand the product in Eq. (3) and separate the dual scalar and the dual-vector part, so that

$$
\begin{array}{r}
\cos \frac{\Delta \psi}{2}-\varepsilon \frac{\Delta t}{2} \sin \frac{\Delta \psi}{2}=\cos \frac{\Delta \theta}{2}-\varepsilon \frac{\Delta d}{2} \sin \frac{\Delta \theta}{2} \mathrm{G} \cdot \mathrm{H} \\
\sin \frac{\Delta \psi}{2}\left(\begin{array}{r}
\tan \frac{\Delta t}{2} \\
1+\varepsilon \frac{\Delta \psi}{\mathrm{RP}}=
\end{array}\right) \sin \frac{\Delta \theta}{2} \mathrm{G}+\varepsilon \frac{\Delta d}{2} \cos \frac{\Delta \theta}{2} \mathrm{H} \\
+\varepsilon \frac{\Delta d}{2} \sin \frac{\Delta \theta}{2} \mathrm{G} \times \mathrm{H}
\end{array}
$$

For studying the screw system, we are only interested in the dual-vector part of Eq. (9), which defines the lines with their associated pitch.

The resulting screw is parametrized by joint variables $\Delta \theta$ of the revolute joint and $\Delta d$ corresponding to the prismatic joint. We denote the expression in Eq. (9) as the finite kinematic generator of the screw system. In the case of finite displacements, the screw systems correspond to subspaces of a projective space, and so we take the unit screws as representatives by dividing the dual vector of Eq. (9) by $\sin (\Delta \psi / 2)$. From the real part of the first equation in Eq. (9), we can see that $\sin (\Delta \psi / 2)= \pm \sin (\Delta \theta / 2)$, so that

$$
\pm\left(1+\varepsilon \frac{\frac{\Delta t}{2}}{\tan \frac{\Delta \psi}{2}}\right) \mathrm{S}_{\mathrm{RP}}=\mathrm{G}+\varepsilon \frac{\frac{\Delta d}{2}}{\tan \frac{\Delta \theta}{2}} \mathrm{H}+\varepsilon \frac{\Delta d}{2} \mathrm{G} \times \mathrm{H}
$$

If we give values to the joint variables $\Delta \theta$ and $\Delta d$, we will generate a screw surface of dimension 3 of a special type, in which all the screws have parallel directions.

It is easy to show that the set of screws generated by the motion of the RP serial chain is indeed a screw system, which is closed under addition and multiplication by scalar. Generate two unit screws $S_{1}$ and $S_{2}$ using Eq. (10) and perform the linear combination with real constants $k_{1}$ and $k_{2}$. We normalize the resulting screw by dividing by $k_{1}+k_{2}$ to obtain

$$
\begin{aligned}
\left(1+\varepsilon \frac{\frac{\Delta t_{3}}{2}}{\tan \frac{\Delta \psi_{3}}{2}}\right) S_{3} & =\frac{1}{k_{1}+k_{2}}\left(k_{1} S_{1}+k_{2} S_{2}\right) \\
& =\mathbf{g}+\varepsilon\left(\mathbf{g}^{0}+\varepsilon \frac{\frac{\Delta d_{c}}{2}}{\tan \frac{\Delta \theta_{c}}{2}} \mathbf{h}+\varepsilon \frac{\Delta d_{c}}{2} \mathbf{g} \times \mathbf{h}\right)
\end{aligned}
$$

where

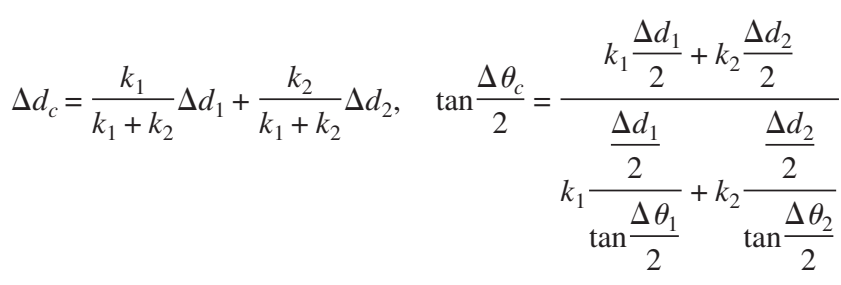

hence the linear combination belongs to the screw system generated by the relative motion of the RP chain. Next is to calculate the dimension of this screw system. Notice that the direction of all unit screws generated is equal to $\mathbf{g}$, and then we just need to look at the dimension of the dual part. Generate three screws with values $\Delta \theta_{i}$ and $\Delta d_{i}, i=1,2,3$. For the three vectors corresponding to the dual part to be linearly independent, the determinant of the column vector matrix must be different from zero. We obtain the value for the determinant

$$
\begin{aligned}
& \operatorname{det}\left(\left[\mathbf{s}_{i}^{0}\right]\right)=\mathbf{g} \cdot \mathbf{h} \quad \mathbf{g}^{0} \cdot \mathbf{h}\left(\frac{\frac{\Delta d_{1}}{2}}{\tan \frac{\Delta \theta_{1}}{2}}\left(\frac{\Delta d_{3}}{2}-\frac{\Delta d_{2}}{2}\right)+\frac{\frac{\Delta d_{2}}{2}}{\tan \frac{\Delta \theta_{2}}{2}}\left(\frac{\Delta d_{1}}{2}\right.\right. \\
&\left.\left.-\frac{\Delta d_{3}}{2}\right)+\frac{\frac{\Delta d_{3}}{2}}{\tan \frac{\Delta \theta_{3}}{2}}\left(\frac{\Delta d_{2}}{2}-\frac{\Delta d_{1}}{2}\right)\right)
\end{aligned}
$$

which is different from zero when the angle $\theta$ and the slide $d$ are independent, except for special parallel or perpendicular arrangements of the joints. Hence, a maximal set of independent screws has cardinality 3 .

In order to generate the screw system of the RPRP linkage, we impose the motion constraint relations between the joint variables. The conditions are derived in Ref. [27] from the closure equations of the linkage, to obtain

$$
\begin{gathered}
d=d_{2} \\
\theta= \pm\left(\theta_{2}-\pi\right)
\end{gathered}
$$




$$
\frac{\tan \frac{\theta}{2}}{d}=\frac{\sin \alpha_{12}}{a_{12} \pm a_{23}}=k
$$

where the twist angle $\alpha_{12}$ and link lengths $a_{12}$ and $a_{23}$ are shown in Fig. 2; the angles $\theta$ and $\theta_{2}$ and slides $d$ and $d_{2}$, also shown in Fig. 2, have to be measured according to the convention [27] from the previous common normal line. The plus/minus sign corresponds to folded and unfolded linkages. For our purposes, only the third condition is needed. It is important to notice that this condition applies to absolute values of the joint variables, measured from the previous common normal line as stated before.

In order to use this relation, substitute $\Delta d=d-d_{0}$ and $\Delta \theta=\theta$ $-\theta_{0}$ to apply Eq. (14) so that the resulting expression depends only on $d$, for instance. For all possible values of the slide $d$, this generates a 2-IB system of screws [31,32]. This can be checked numerically by generating six random screws and computing the rank of the matrix that has the screws as columns. In this case, the rank is 2. It can also be shown that when the third condition in Eq. (14) holds, the determinant in Eq. (13) is equal to zero.

The unit screws of the system can be related to spatial displacements if we add the value of the magnitude of the screw. For finite displacement screws, the value of the magnitude related to each screw is unique; this is due to the fact that finite-screw systems are projective subspaces [33]. The information about the magnitude corresponding to each screw can be extracted from the scalar part of the dual quaternion product in Eq. (9). This equation relates the rotation associated to the resulting screw, $\Delta \psi$, to the joint variables of the kinematic chain. For the RPRP chain, this yields $\sin \Delta \psi / 2= \pm \sin \Delta \theta / 2$ as noted before.

Figure 3 illustrates the nonlinear relation between the screws generated by an RPRP closed chain and the corresponding set of absolute positions of the end-effector (for those positions we assume that the reference configuration is the identity). We use as values for the linkage those of an example from Ref. [27].

3.2 The Finite-Screw System Generated as a Linear Combination of Two Screws. It has been shown that the relative displacements of the RPRP chain generate a 2-IB finite-screw system. By definition, this same screw system can be generated as the linear combination of two screws with same direction and arbitrary location and (possibly) finite pitches. From a synthesis point of view, the key is that this coincides with the results of the counting in Sec. 2.2. The task positions defined for the synthesis of the $\mathrm{RP}$ (or PR) chain are two relative displacements with same direction and, in general, finite pitches.

The screw system is characterized by the pitch distribution as a linear function of the distance between screws along a common normal. In this derivation, we can parameterize the results as a function of the angle $\theta$ or the slide $d$.

Consider the screw $S_{\mathrm{RP}}=(1+\varepsilon p) \mathrm{S}_{\mathrm{RP}}=\mathbf{s}_{\mathrm{RP}}+\varepsilon \mathbf{s}_{\mathrm{RP}}^{0}$. The pitch is obtained by computing $p=\mathbf{s}_{\mathrm{RP}} \cdot \mathbf{s}_{\mathrm{RP}}^{0} / \mathbf{s}_{\mathrm{RP}} \cdot \mathbf{s}_{\mathrm{RP}}$ in Eq. (10),

$$
p=\frac{1+k^{2} d d_{0}}{2 k} \mathbf{g} \cdot \mathbf{h}=\frac{1}{2 k}\left(1+\tan \frac{\theta_{0}}{2} \tan \frac{\theta}{2}\right) \mathbf{g} \cdot \mathbf{h}
$$

For two screws $S_{A}$ and $S_{B}$, the difference in the pitches is given by

$$
p_{B}-p_{A}=\frac{k d_{0} \mathbf{g} \cdot \mathbf{h}}{2}\left(d_{B}-d_{A}\right)=\frac{\tan \frac{\theta_{0}}{2} \mathbf{g} \cdot \mathbf{h}}{2 k}\left(\tan \frac{\theta_{B}}{2}-\tan \frac{\theta_{A}}{2}\right)
$$

The distance between two screws along the common normal is calculated by finding the perpendicular point on the axis, $\mathbf{c}_{\mathrm{RP}}$ $=\left(\mathbf{s}_{\mathrm{RP}} \times \mathbf{s}_{\mathrm{RP}}^{0}\right) /\left(\mathbf{s}_{\mathrm{RP}} \cdot \mathbf{s}_{\mathrm{RP}}\right)$ and computing the norm of the difference for two of them,
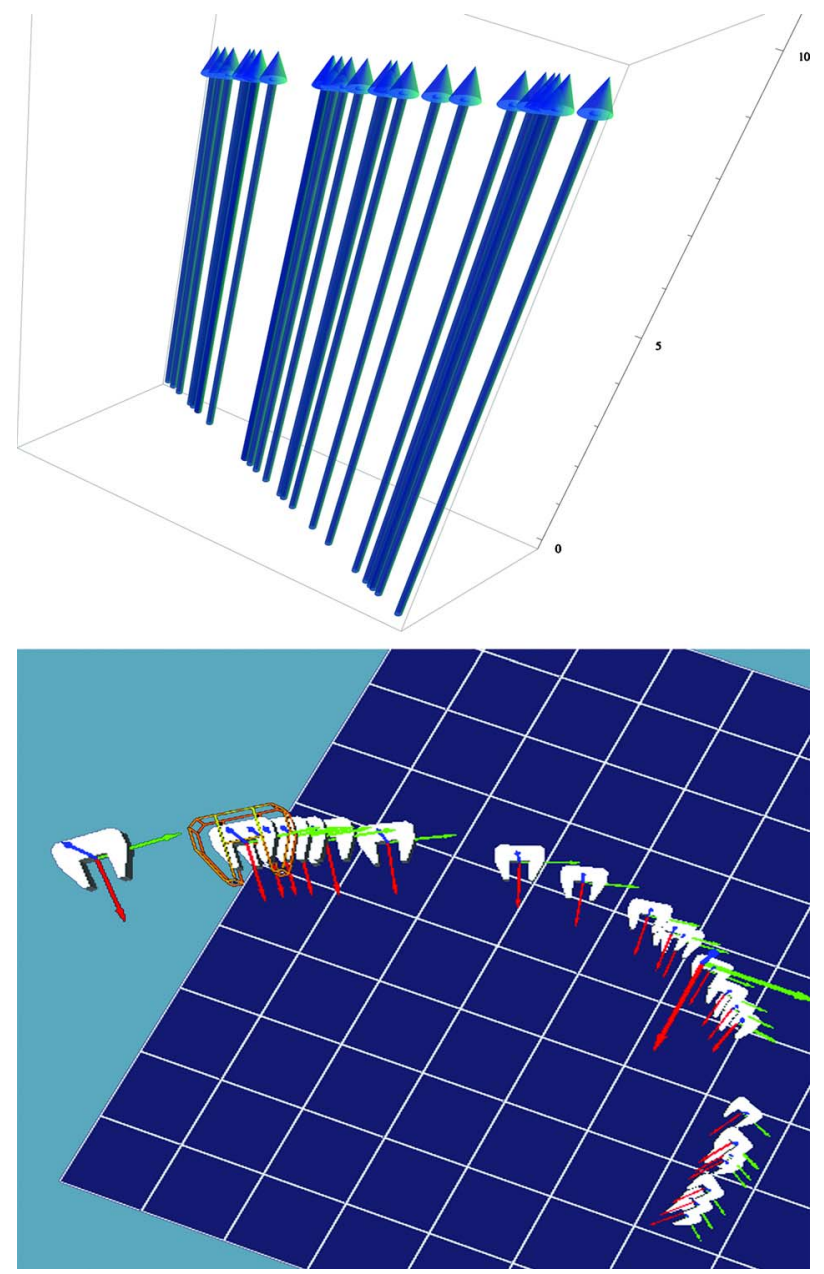

Fig. 3 Screw system generated by the RPRP linkage, above; corresponding absolute displacements, below

$$
\begin{aligned}
\mathbf{c}_{B}-\mathbf{c}_{A} & =\left(\frac{k d_{0}}{2} \mathbf{g} \times \mathbf{h}+\frac{1}{2}(\mathbf{g g} \cdot \mathbf{h}-\mathbf{h})\right)\left(d_{B}-d_{A}\right) \\
& =\frac{1}{2 k}\left(\tan \frac{\theta_{0}}{2} \mathbf{g} \times \mathbf{h}+\mathbf{g g} \cdot \mathbf{h}-\mathbf{h}\right)\left(\tan \frac{\theta_{B}}{2}-\tan \frac{\theta_{A}}{2}\right)
\end{aligned}
$$

and

$$
\begin{aligned}
\left\|\mathbf{c}_{B}-\mathbf{c}_{A}\right\| & =\frac{1}{2}\left|d_{B}-d_{A}\right| \sqrt{\sin ^{2} \alpha_{12}\left(1+d_{0}^{2} k^{2}\right)} \\
& =\frac{1}{2}\left|\left(\tan \frac{\theta_{B}}{2}-\tan \frac{\theta_{A}}{2}\right)\right| \sqrt{\frac{\sin ^{2} \alpha_{12}}{k^{2} \cos ^{2} \frac{\theta_{0}}{2}}}
\end{aligned}
$$

The slope of the (linear) pitch distribution is computed as

$$
K=\frac{p_{B}-p_{A}}{\left\|\mathbf{c}_{B}-\mathbf{c}_{A}\right\|}
$$

and for the RPRP chain we can simplify the expression to

$$
K= \pm \frac{\cos \alpha_{12}}{\sqrt{d_{0}^{2} \sin ^{2} \alpha_{12}+\left(a_{12}+a_{23}\right)^{2}}} d_{0}= \pm \frac{\cos \alpha_{12}}{\sin \alpha_{12}} \sin \frac{\theta_{0}}{2}
$$


Notice that the slope is constant and depends on the initial configuration. The sign is negative if $d_{B}<d_{A}$ or if $\tan \theta_{B} / 2$ $<\tan \theta_{A} / 2$.

The screw system is related to the displacement of the RPRP linkage by computing the distribution of the magnitude with respect to the known pitch, distance, and magnitude of the screws $S_{A}$ and $S_{B}$ used to define the system. Consider the magnitude of the screws as $m=\sin (\Delta \psi / 2)= \pm \sin (\Delta \theta / 2)$. Knowing the magnitude and pitch of screws $S_{A}$ and $S_{B}$, we can solve for the magnitude $m_{C}$ of a screw $S_{C}$ using Eq. (18),

$$
m_{C}= \pm \sin \frac{\Delta \theta_{C}}{2}=\frac{X}{\sqrt{1+X^{2}}}
$$

where the factor $X$ is simplified using Eqs. (15)-(20) to

$$
X=\frac{\left\|\mathbf{c}_{B}-\mathbf{c}_{A}\right\| p_{A} \tan \frac{\Delta \theta_{A}}{2}-\left\|\mathbf{c}_{C}-\mathbf{c}_{A}\right\|\left(p_{A} \tan \frac{\Delta \theta_{A}}{2} \pm p_{B} \tan \frac{\Delta \theta_{B}}{2}\right)}{\left\|\mathbf{c}_{B}-\mathbf{c}_{A}\right\| p_{C}}
$$

The positive and negative signs in the expression of $X$ correspond to the sign of the $\tan \left(\theta_{B} / 2\right)-\tan \left(\theta_{A} / 2\right)$ factor.

3.3 Defining the Kinematic Task as a Finite-Screw System. It is now possible to define the screw system as the input task for the dimensional synthesis of the RPRP closed chain. As a linear subspace, it is easy to shape the task at our convenience. Once we have a satisfactory screw system, any two screws from it can be selected to perform the dimensional synthesis and to obtain a finite number of solutions (in this case, just one).

We have several strategies for shaping the screw system. For instance, we can define a first relative displacement, $\hat{S}_{12}$ $=\cos \left(\widehat{\Delta \psi_{12}} / 2\right)+\sin \left(\widehat{\Delta \psi_{12}} / 2\right)\left(\mathbf{s}_{12}+\varepsilon \mathbf{s}_{12}^{0}\right)$. The rotation axis of the displacement, $\mathbf{s}_{12}$, is common to both $\hat{S}_{12}$ and the second relative displacement. We set $\mathbf{s}_{12}=\mathbf{s}_{13}$ and select a rotation angle to define the relative rotation $\hat{s}_{13}$.

We can then set the slope of the pitch distribution in order to shape the screw system. The pitch for the finite displacement screws is [20]

$$
p_{1 i}=\frac{\frac{\Delta t_{1 i}}{2}}{\tan \frac{\Delta \psi_{1 i}}{2}}
$$

directly calculated from the dual quaternion using $p_{1 i}$ $=\mathbf{s}_{1 i} \cdot \mathbf{s}_{1 i}^{0} / \mathbf{s}_{1 i} \cdot \mathbf{s}_{1 i}$. Similarly, a point on the screw axis is calculated as

$$
\mathbf{c}_{1 i}=\frac{\mathbf{s}_{1 i} \times \mathbf{s}_{1 i}^{0}}{\mathbf{s}_{1 i} \cdot \mathbf{s}_{1 i}} .
$$

Define the slope of the distribution as $K=\left(p_{13}-p_{12}\right) /\left(\left\|\mathbf{c}_{13}-\mathbf{c}_{12}\right\|\right)$, according to Eq. (20). If we set the value of $K$, we can solve for $\Delta t_{13}$ in order to define the pitch of the second relative displacement, the location of its screw axis being defined. This is one possible way of defining the screw system. Converting from this to absolute displacements, we can easily check whether the trajectory for the synthesis is acceptable.

Any other strategy to basically define a triangle in space can be used. The purpose of this is to have a better control on the shape of the trajectory of the linkage than the one given by just two separate finite positions.

\section{Dimensional Synthesis of the RPRP Linkage for a Prescribed Screw System}

The synthesis of the RP, and similarly, PR chains, is simple and yields one solution. In general, the task positions are expressed as
Table 1 Goal relative displacements for the RP and PR chains, Huang's example

$(0,0,-0.05,0.99)+\varepsilon(0.02,0.37,-0.51,-0.03)$

$(0,0,-0.34,0.94)+\varepsilon(0.80,2.23,-3.08,-1.10)$

relative displacements $\hat{Q}_{1 i}=\hat{Q}_{i} \hat{Q}_{1}^{-1}$ with respect to the first task position $\hat{Q}_{1}$. For the RP and PR chains, we can define up to two relative task positions, as explained in Sec. 2.2. Given an arbitrary relative displacement $\hat{Q}_{12}=\left(q_{12}^{w}+\mathbf{q}_{12}\right)+\varepsilon\left(q_{12}^{w 0}+\mathbf{q}_{12}^{0}\right)$ and a second displacement $\hat{Q}_{13}=\left(q_{13}^{w}+\mathbf{q}_{13}\right)+\varepsilon\left(q_{13}^{w 0}+\mathbf{q}_{13}^{0}\right)$ such that both have same direction and a given pitch distribution, we equate them to the forward kinematics in Eq. (3). We can solve for the direction of the revolute joint $\mathbf{g}$ and the rotation angles,

$$
\mathbf{g}=\frac{\mathbf{q}_{12}}{\left\|\mathbf{q}_{12}\right\|}, \quad \tan \frac{\Delta \theta_{1 i}}{2}=\frac{\left\|\mathbf{q}_{1 i}\right\|}{q_{1 i}^{w}}, \quad i=2,3
$$

The equations corresponding to the dual part are linear in the moment of the revolute joint, $\mathbf{g}^{0}$,

$$
\mathbf{g}^{0}=\frac{1}{\sin \frac{\Delta \theta_{1 i}}{2}}\left(\mathbf{q}_{1 i}^{0}-\frac{\Delta d_{1 i}}{2}\left(\cos \frac{\Delta \theta_{1 i}}{2} \mathbf{h}+\sin \frac{\Delta \theta_{1 i}}{2} \mathbf{g} \times \mathbf{h}\right)\right), \quad i=1,2
$$

Equating the solution of $\mathbf{g}^{0}$ for both relative displacements, we can solve linearly for $\mathbf{h}$ as a function of the slides $\Delta d_{12}$ and $\Delta d_{13}$. The relation between the slides is given by the pitch condition,

$$
\frac{q_{12}^{w 0}}{\frac{\Delta d_{12}}{2} \sin \frac{\Delta \theta_{12}}{2}}=\frac{q_{13}^{w 0}}{\frac{\Delta d_{13}}{2} \sin \frac{\Delta \theta_{13}}{2}}
$$

Imposing $\|\mathbf{h}\|=1$, we can solve for the slides to obtain one solution. Using the same process, we can solve for the PR serial chain.

\section{Examples}

Two examples are presented below. The first one is performed to check the method, while for the second one, a fully general task is used.

5.1 First Example. In this first case, the two relative displacements are generated using the RP chain presented in Ref. [27]. In this example, the revolute joint is located at the origin and the prismatic joint is located along the $x$ direction with a twist angle of $\alpha_{12}=\pi / 5$ and link length $a_{12}=5$. We use the loop condition in Eq. (14) and random values for the slide to generate the set of relative displacements of the corresponding RPRP closed chain. From those, we randomly select two displacements, the ones in Table 1.

The resulting screw system is shown in Fig. 4, where the length of each screw is proportional to its pitch. Also the corresponding trajectory of absolute displacements, considering the reference position as the identity, is shown in the same figure.

Using the synthesis procedure explained in Sec. 4, we obtain the RP and PR chains of Table 2. Notice that they coincide with the values given in Ref. [27], which means this is a folded RPRP overconstrained movable linkage. Figure 5 shows the linkage reaching some of the positions. Notice that the shape of the coupler link attached to the end-effector depends on the position chosen as reference configuration. 


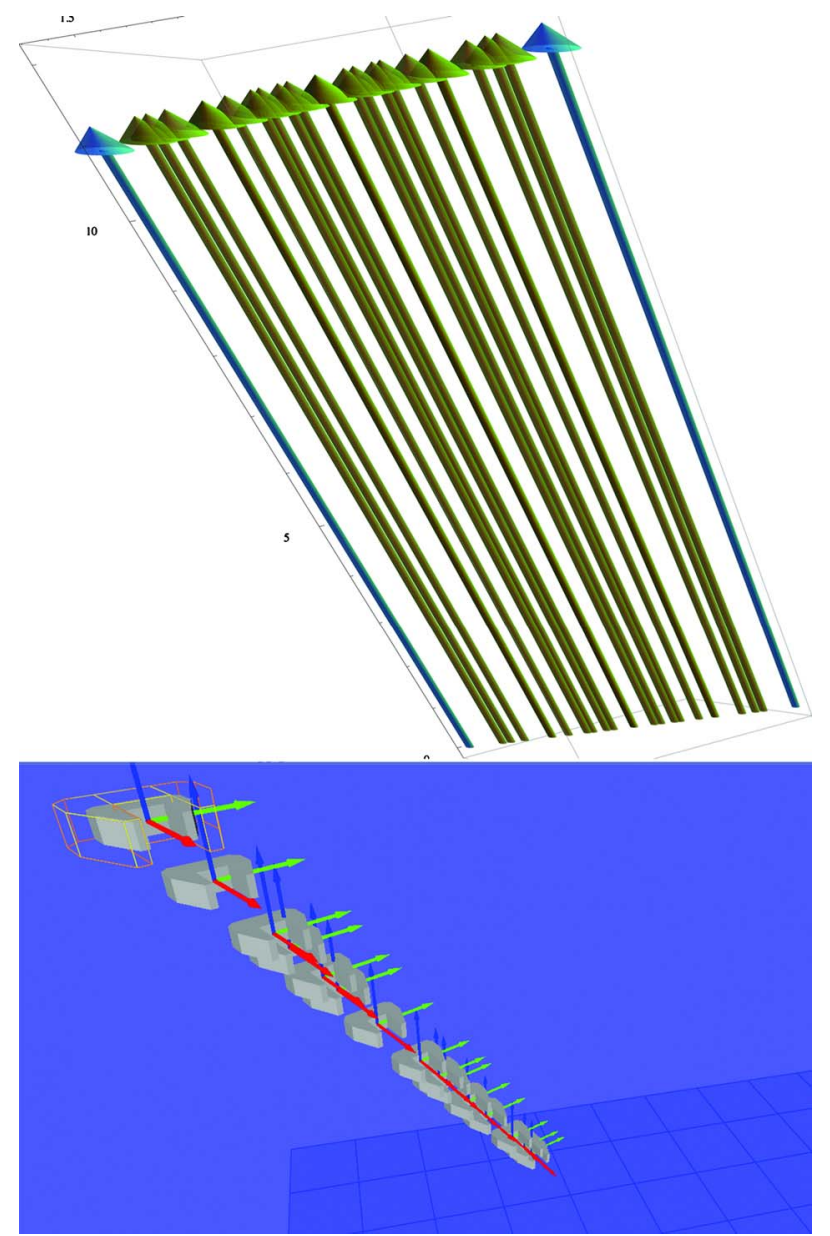

Fig. 4 Above, screw system generated by $S_{12}$ and $S_{13}$ (shown as first and last screws); below, corresponding absolute displacements with reference displacement being the identity

5.2 Second Example. For the second example, the dual quaternions in Table 3 have been generated as explained. $\hat{S}_{12}$ has been randomly generated, while the rotation in $\hat{S}_{13}$ is such that it belongs to the workspace of the chain.

We set the location of the second screw axis of the relative displacement with a point $\mathbf{p}_{13}=(0.083,2.159,-3.226)$, randomly generated. Then we choose a value for the slope of the pitch distribution, $K=0.480$, which allows us to create the second rela-

Table 2 Joint axes for the RPRP linkage at the reference configuration, example 1

\begin{tabular}{lcc}
\hline \hline Chain & Revolute joint $\mathbf{G}$ & Prismatic joint $\mathbf{h}$ \\
\hline RP axes & $\left\{\begin{array}{l}0+0 \varepsilon \\
0+0 \varepsilon \\
1+0 \varepsilon\end{array}\right\}$ & $\left\{\begin{array}{c}0 \\
0.59, \\
-0.81\end{array}\right\}$ \\
RP joint vars. & $\theta_{12}=-5.7$ & $d_{12}=1.27$ \\
$\theta_{13}=-39.4$ & $d_{13}=8.08$ \\
\hline & $\left\{\begin{array}{c}0+5.38 \varepsilon \\
0+13.0 \varepsilon \\
-1+0 \varepsilon\end{array}\right\}$ & $\left\{\begin{array}{l}-0.42 \\
-0.42 \\
\text { PR axes }\end{array}\right.$ \\
& $\theta_{12}=5.7$ & $d_{12}=1.27$ \\
PR joint vars. & $\theta_{13}=39.4$ & $d_{13}=8.08$ \\
\hline \hline
\end{tabular}

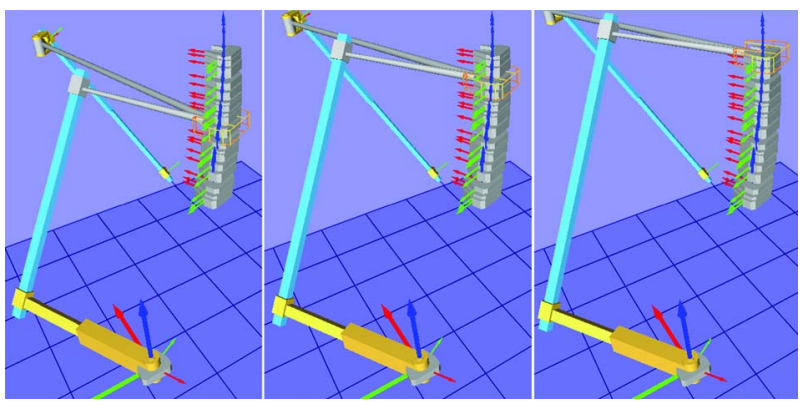

Fig. 5 RPRP linkage reaching three positions of the trajectory

tive displacement.

The resulting screw system is shown in Fig. 6, where the length of each screw is proportional to its pitch. The corresponding trajectory of absolute displacements, considering the reference position as the identity, is shown in Fig. 7.

We obtain one solution for the RPRP linkage, specified in Table 4 as the Plücker coordinates of the axes and the joint variables to reach the positions.

Again, the dimensions form an overconstrained movable RPRP linkage. Figure 8 shows the chain reaching three displacements along the trajectory, considering the reference displacement as the identity.

\section{Conclusions}

This paper presents the exact workspace synthesis of an overconstrained closed linkage, the RPRP. The knowledge of the screw system that corresponds to the finite displacements of the linkage is used to generate the workspace of the linkage, which in turn ensures that the solutions of the synthesis of the RP and PR serial chains can be assembled to create a movable system. The counting of the maximum number of positions for the finite-

Table 3 Goal relative displacements for the RP and PR chains

$(0.46,-0.13,-0.56,-0.67)+\varepsilon(1.66,0.34,-0.02,1.08)$

$(0.13,-0.04,-0.17,0.98)+\varepsilon(0.02,-0.57,-0.92,-0.18)$

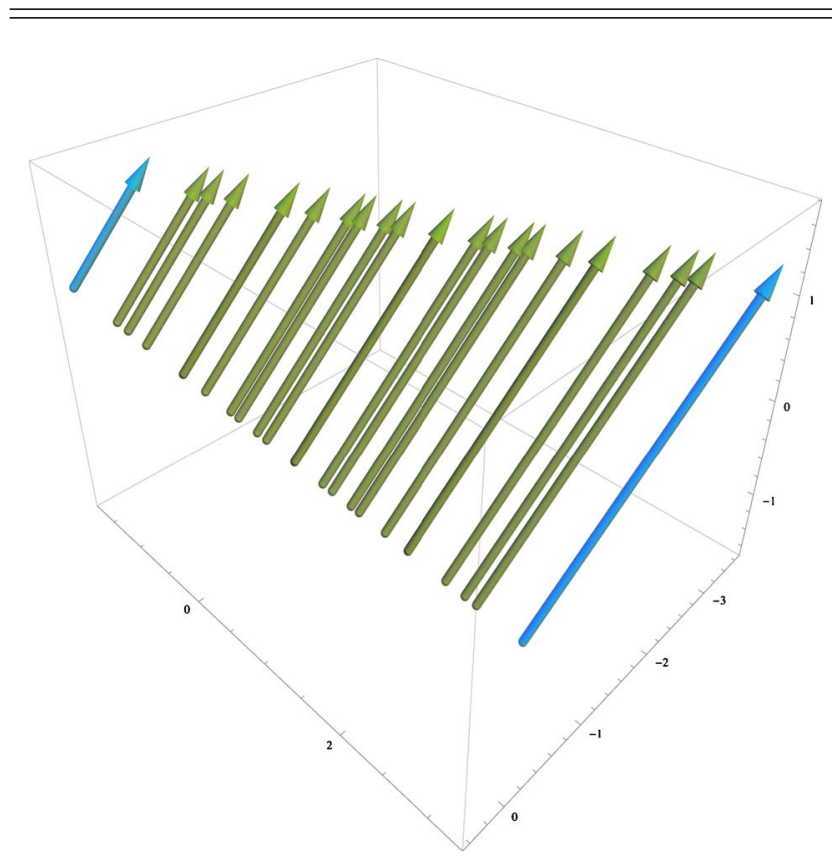

Fig. 6 Screw system generated by $S_{12}$ and $S_{13}$ (first and last screws) 


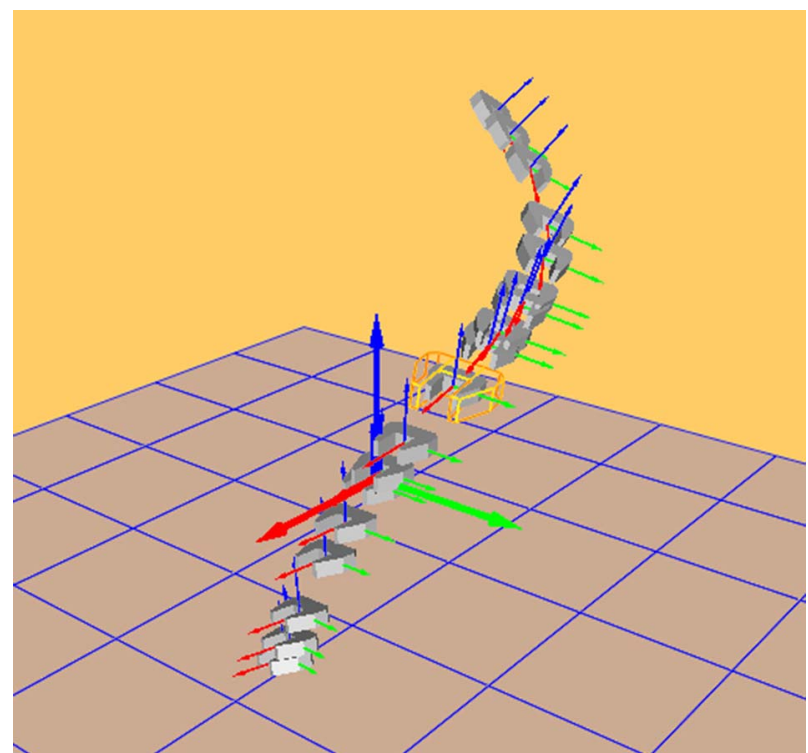

Fig. 7 The RPRP end-effector task trajectory

Table 4 Joint axes for the RPRP linkage at the reference configuration, example 2

\begin{tabular}{lcc}
\hline \hline Chain & Revolute joint $\mathrm{G}$ & Prismatic joint $\mathbf{h}$ \\
\hline RP axes & $\left\{\begin{array}{c}-0.62-0.44 \varepsilon \\
0.18-3.17 \varepsilon \\
0.76+0.39 \varepsilon\end{array}\right\}$ & $\left\{\begin{array}{c}-0.09 \\
0.89 \\
0.44\end{array}\right\}$ \\
$\theta_{12}=-264.5$ & $d_{12}=5.30$ \\
$\theta_{13}=-25.2$ & $d_{13}=-3.05$ \\
RP joint vars. & $\left\{\begin{array}{c}0.62+2.32 \varepsilon \\
-0.18-1.83 \varepsilon \\
-0.76+2.31 \varepsilon \\
\theta_{12}=264.5 \\
\theta_{13}=25.2\end{array}\right.$ \\
\hline PR axes & $\left\{\begin{array}{c}-0.27 \\
0.06 \\
-0.96\end{array}\right\}$ \\
PR joint vars.
\end{tabular}

position synthesis of the serial chain turns out to fully define the finite-screw system of the linkage. This provides an easy method to shape the whole trajectory of the RPRP linkage as an input for the synthesis process. Even though the method targets the whole motion of the linkage, the synthesis equations need to be stated only at the task positions used to generate the linear combination of screws; hence, the finite-position synthesis equations can still be used. The method yields a single RPRP linkage.

\section{Acknowledgment}

This work is supported by a Ramon y Cajal Research Fellowship from the Spanish Ministry of Science and Innovation.

\section{Nomenclature}

$$
\begin{aligned}
S= & \text { line or dual vector, defined using Plücker } \\
& \text { coordinates. } \\
\mathbf{S}= & \text { vector } \\
\hat{S}= & \text { quaternion or dual quaternion } \\
\hat{w}= & \text { dual number } \\
{[M]=} & \text { matrix }
\end{aligned}
$$
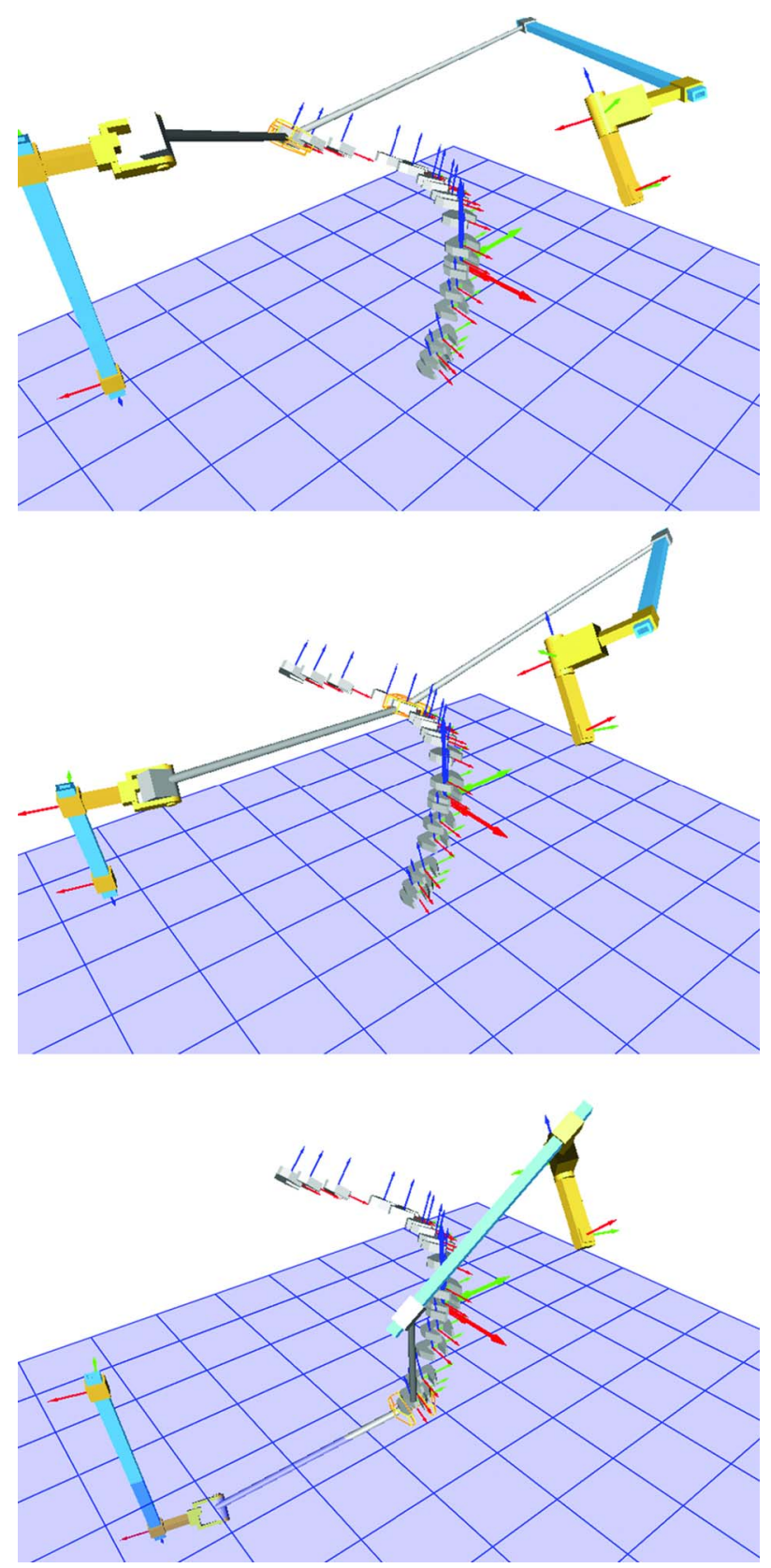

Fig. 8 The RPRP linkage reaching three positions along the trajectory

\section{References}

[1] Hervé, J. M., 1999, “The Lie Group of Rigid Body Displacements, a Fundamental Tool for Mechanism Design," Mech. Mach. Theory, 34, pp. 719-730.

[2] Angeles, J., 2002, "The Qualitative Synthesis of Parallel Manipulators," Proceedings of the Workshop on Fundamental Issues and Future Research Directions for Parallel Mechanisms and Manipulators, C. Gosselin and I. EbertUphoff, eds.

[3] Gogu, G., 2007, Structural Synthesis of Parallel Robots. Part 1: Methodology, 1 st ed., Springer, New York.

[4] Huang, T., Li, M., Zhao, X. M., Mei, J., Chetwynd, D. G., and Hu, S. J., 2005, "Conceptual Design and Dimensional Synthesis for a 3-dof Module of the Trivariant-A Novel 5-dof Reconfigurable Hybrid Robot," IEEE Trans. Rob. Autom., 21(3), pp. 449-456.

[5] Huang, T., Zhanxian, L., Li, M., Chetwynd, D. G., and Gosselin, C. M., 2004, "Conceptual Design and Dimensional Synthesis of a Novel 2-dof Translational Parallel Robot for Pick-and-Place Operations," AMSE J. Mech. Des., 126, pp. 449-455.

[6] Kim, H. S., and Tsai, L.-W., 2003, "Design Optimization of a Cartesian Parallel Manipulator,” ASME J. Mech. Des., 125, pp. 43-51.

[7] Affi, Z., Romdhane, L., and Maalej, A., 2004, "Dimensional Synthesis of a 
3-Translational-dof in-Parallel Manipulator for a Desired Workspace,” Eur. J. Mech. A/Solids, 23, pp. 311-324.

[8] Kosinska, A., Galicki, M., and Kedzior, K., 2003, "Design and Optimization of Parameters of Delta-4 Parallel Manipulator for a Given Workspace," J. Rob. Syst., 20(9), pp. 539-548.

[9] Chablat, D., and Wenger, P., 2003, "Architecture Optimization of a 3-dof Parallel Mechanism for Machining Applications, the Orthoglide," IEEE Trans. Robot. Autom., 19(3), pp. 403-410.

[10] Merlet, J.-P., 2005, "Optimal Design of Robots," Proceedings of Robotics: Science and Systems, Cambridge, USA.

[11] Wolbrecht, E., Su, H.-J., Perez, A., and McCarthy, J. M., 2004, "Geometric Design of Symmetric 3-rrs Constrained Parallel Platforms," Proceedings of the 2004 ASME International Mechanical Engineering Congress and Exposition, ASME, New York.

[12] Kim, H. S., and Tsai, L.-W., 2003, "Kinematic Synthesis of a Spatial 3-rps Parallel Manipulator," ASME J. Mech. Des., 125, pp. 92-97.

[13] Rao, N. M., and Rao, K. M., 2009, "Dimensional Synthesis of a 3-rps Parallel Manipulator for a Prescribed Range of Motion of Spherical Joints," Mech. Mach. Theory, 44, pp. 477-486.

[14] Ravani, B., and Roth, B., 1983, "Motion Synthesis Using Kinematic Mappings," ASME J. Mech., Transm., Autom. Des., 105(3), pp. 460-467.

[15] Hayes, M. J. D., Luu, T., and Chang, X. W., 2004, "Kinematic Mapping Application to Approximate Type and Dimension Synthesis of Planar Mechanisms," On Advances in Robot Kinematics, J. Lenarcic and C. Galletti, eds., Kluwer, Dordrecht

[16] Schröcker, H.-P., Husty, M. L., and McCarthy, J. M., 2007, "Kinematic Mapping Based Assembly Mode Evaluation of Planar Four-Bar Mechanisms," ASME J. Mech. Des., 129, pp. 924-929.

[17] Wu, J., Purwar, A., and Ge, Q. J., 2010, "Interactive Dimensional Synthesis and Motion Design of Planar $6 R$ Single-Loop Closed Chains via Constrain Manifold Modification,” ASME J. Mech. Rob., 2(3), p. 031012.

[18] Husty, M. L., Pfurner, M., Schrocker, H.-P., and Brunnthaler, K., 2007, "Algebraic Methods in Mechanism Analysis and Synthesis," Robotica, 25, pp. 661-675.

[19] Brunnthaler, K., 2006, "Synthesis of 4r Linkages Using Kinematic Mapping," Ph.D. thesis, University of Innsbruck, Innsbruck, Austria.
[20] Parkin, I. A., 1992, "A Third Conformation With the Screw Systems: Finite Twist Displacements of a Directed Line and Point," Mech. Mach. Theory, 27, pp. $177-188$.

[21] Huang, C., 1994, "On the Finite Screw System of the Third Order Associated With a Revolute-Revolute Chain," ASME J. Mech. Des., 116, pp. 875-883.

[22] Huang, C., 1996, "The Cylindroid Associated With Finite Motions of the Bennett Mechanism," Proceedings of the ASME Design Engineering Technical Conferences, Irvine, CA, August.

[23] Baker, J. E., 1998, "On the Motion Geometry of the Bennett Linkage," Proceedings of the Eighth International Conference on Engineering Computer Graphics and Descriptive Geometry, pp. 433-437.

[24] Perez, A., and McCarthy, J. M., 2003, "Dimensional Synthesis of Bennett Linkages," ASME J. Mech. Des., 125(1), pp. 98-104.

[25] Waldron, K. J., 1973, "A Study of Overconstrained Linkage Geometry by Solution of Closure Equations-Part II-Four-Bar Linkages With Lower Pair Joints Other Than Screw Joints," Mech. Mach. Theory, 8, pp. 233-247.

[26] Baker, J. E., 1975, "The Delassus Linkages," Proceedings of the Fourth World Congress on the Theory of Machines and Mechanisms, p. 4549.

[27] Huang, C., 2006, "Linear Property of the Screw Surface of the Spatial rprp Linkage," ASME J. Mech. Des., 128, pp. 581-586.

[28] Perez-Gracía, A., and McCarthy, J. M., 2006, "The Kinematic Synthesis of Spatial Serial Chains Using Clifford Algebra Exponentials," Proc. Inst. Mech. Eng., Part C: J. Mech. Eng. Sci., 220(7), pp. 953-968.

[29] Lee, E., and Mavroidis, C., 2002, "Solving the Geometric Design Problem of Spatial 3r Robot Manipulators Using Polynomial Homotopy Continuation,' ASME J. Mech. Des., 124(4), pp. 652-661.

[30] Perez, A., and McCarthy, J. M., 2004, "Dual Quaternion Synthesis of Constrained Robotic Systems," ASME J. Mech. Des., 126(3), pp. 425-435.

[31] Hunt, K. H., 1978, Kinematic Geometry of Mechanisms, Oxford University Press, New York.

[32] Zlatanov, D., Agrawal, S., and Gosselin, C. L., 2005, "Convex Cones in Screw Spaces," Mech. Mach. Theory, 40, pp. 710-727.

[33] Huang, C., Sugimoto, K., and Parkin, I., 2008, "The Correspondence Between Finite Screw Systems and Projective Spaces," Mech. Mach. Theory, 43, pp. $50-56$ 